\title{
The development of the sector risk profiling methodology for Australian civil aviation activity and its application to the small aeroplane transport sector
}

\author{
$\underline{\text { X.G. Lin }}^{\text {a }}$, F. Fernandes ${ }^{\text {a }}$, S.M. Duffield ${ }^{\mathrm{b}}$ and J. Codyre ${ }^{\mathrm{a}}$ \\ ${ }^{a}$ Intelligence and Analytics Section, Strategy, Quality \& Intelligence Branch, Civil Aviation Safety Authority, \\ Australian Capital Territory \\ Email:xunguo.lin@casa.gov.au \\ ${ }^{b}$ Retired at BOGANGAR 2488, New South Wales, sduffiel@optusnet.com.au
}

\begin{abstract}
Sector Risk Profiling (SRP) is a methodology to develop a risk picture for a sector of civil aviation activity using ISO 31000:2009 Risk Management: Principles and guidelines. By engaging with sector stakeholders to consult and review all available information on the sector to develop the risk picture, optimal risk responses for risks of significance can be collectively identified and ownership assigned for implementing responses.
\end{abstract}

Although the SRP methodology is by large a qualitative approach, quantitative data analysis can be integrated into the SRP if relevant data is available. The SRP offers four advantages: (1) It is consultation driven by sector's subject matter experts; (2) It produces a sector risk register which includes all risk attributes such as causes, current controls, future treatments, ownerships, etc.; (3) It suggests an assurance mapping process which contains a gap analysis of the sector risk register and each sector entity's risk register; and (4) It introduces a new concept of the "living risk profile" by implementing and integrating the risk register into the sector entity's Safety Management System (SMS) and into the authority's safety surveillance program to responding to emerging risks.

The SRP process contains three phases. Phase 1 establishes the sector context to assist data collection and analysis. An industry workshop is conducted towards the end of Phase 1. The workshop participants include sector entities (operators), safety authority and other sector stakeholders providing infrastructure and support services. The key outputs from the workshop are: a set of sector objectives, SWOT (Strengths, Weaknesses, Opportunities and Threats) analysis to identify the hazards and associated risks for the sector.

The principal task in Phase 2 is to develop a sector risk register based on the hazards and associated risks at the second workshop. During the workshop the participants engage in a critical assessment of hazards to develop risk statements, impacts from the risks, identify current risk controls and assess current risks using a risk matrix and the developed sector objectives. The information captured in the risk register include causes, impacts, existing controls, current likelihood, consequence and risk rating. When the current risk rating is outside the ALARP (As Low As Reasonably Practicable) limits, additional risk treatments are also identified with accountability assigned to owners best placed to treat the risk. The residual risk is assessed assuming treatments are in place. As part of the collaboration and engagement, the risk register is revised within the workshop participants.

Phase 3 consists of sector assurance mapping, including conducting a gap analysis between the sector risk register and each operator's risk register. This assurance mapping contributes to the authority's sector surveillance program, including the development of Safety Performance Indicators (SPIs) specifically for the sector. Phase 3 also integrates the sector risk register into sector operator's SMS and into the authority's sector surveillance program, hence, forms a living risk profiling process to evaluating the current risk controls and responding to changing operational environment and emerging risks.

The way forward in the 'SRP space' is to 'collaborate more' and 'engage more' with all stakeholders to increase understanding, promote safety improvement opportunities, and work on solutions together. This is underpinned by creating a culture that encourages open and honest communication, listening to others, showing respect and maintaining trust.

The SRP methodology will be demonstrated for a sector in air transport. However, SRP methodology is not limited to aviation, it can be applied to any industry sector.

Keywords: $\quad$ Sector risk profile, safety regulation, risk management, risk assessment, risk analysis

(C) CASA 2017 The paper is provided under the Creative Commons Attribution 4.0 International licence, with the exception of CASA's logo, any content supplied by third parties, and other materials specifically not provided under a Creative Commons Attribution 4.0 licence. 


\section{INTRODUCTION}

In recent times, it is common practice to adopt risk management techniques to almost any industry or company (Chapman, 2011). This paper introduces a Sector Risk Profiling (SRP) methodology which adopts the ISO 31000:2009 Risk Management: Principles and guidelines at a sector level in the aviation industry. The concept of sector in this paper is a collection of organisations (aircraft operators in the aviation environment) supplying similar services or producing similar goods. The sector definition used in this paper differs from that adopted in academic literature as a concept introduced at industry level such as the energy sector or housing sector (UK, 2016). The sector concept advanced in the paper requires the organisations within the sector to have a high degree of homogeneity which ensures consistency in a sector-wide risk management.

SRP is a process of developing a risk picture specific to a sector by assembling all available information on the sector in terms of its characteristics and exposure to unsafe events, estimating the likelihood and severity of the event outcomes under the current risk controls and crafting optimal risk responses including additional risk treatments. The SRP process is conducted in three stages uses formal consultations with Subject Matter Experts (SMEs) and sector participants. SRP process supplements the safety risk management function of an aviation safety authority.

The SRP supports Australia's Aviation State Safety Program (Infrastructure, 2016) and can play an important role in transitioning CASA from a compliance based approach to a performance based approach to regulation. The SRP methodology has been successfully applied to many Australia's civil aviation sectors in the past several years and a list of SRPs can found in https://www.casa.gov.au/about-us/standardpage/research-and-statistics. The Civil Aviation Authority New Zealand has also adopted the SRP methodology and practice and recently undertook a SRP for large and medium aeroplanes transport sector (https://www.caa.govt.nz/safety-info/safety-reports/sector-risk-profiles/). Note that SRP methodology is not limited to aviation, it can be applied to any sector.

Although the SRP methodology is by large a qualitative approach, quantitative data analysis can be integrated into the SRP if relevant data is available. The SRP offers four advantages: (1) It is consultation driven by sector's subject matter experts; (2) It produces a sector risk register which includes all risk attributes such as causes, current controls, future treatments, ownerships, etc.; (3) It suggests an assurance mapping process which contains a gap analysis of the sector risk register and each sector entity's risk register; and (4) It introduces a new concept of the "living risk profile" by implementing and integrating the risk register into the sector entity's Safety Management System (SMS) and into the authority's safety surveillance program to responding to emerging risks.

The remainder of this paper is organized into the following sections: Section 2 describes the proposed SRP methodology and process. Section 3 demonstrates an application of the SRP process to the small aeroplane transport sector. Section 4 concludes the paper.

\section{SRP METHODOLOGY AND PROCESS}

\subsection{SRP Methodology}

The SRP methodology is an evidence-based approach based on an analysis of all safety related data sources and industry surveys along with SMEs collaborating in industry workshops. The analytical outputs) are used to support the process in the workshop but. The SRP methodology can be applied to industries where data might be incomplete or unavailable. Furthermore, SRP incorporates the implementation and integration of the sector risk register into an operator's risk register and SMS and the authority's safety assurance and surveillance programs.

The key characteristics of a SRP is that is forward-looking, engages with sector participants and is collaborative and provides an opportunity for industry and the authority to work as a team. The SRP methodology uses the following elements to develop a profile:

- Data fusion to build relationships, patterns and transformations with data,

- Team formation from functional areas within the authority which regulates/oversights the industry,

- Workshops to develop a sector risk register along with safety initiatives if required,

- Monitoring the safety performance of the sector using Safety Performance Indicators (SPI), and

- Exchange of safety performance information with sector entities to update and review the profile.

The application of the SRP process requires a definition for the sector under study based on its activity. Data that is relevant and appropriate is obtained from several sources, and is supplemented by surveys conducted 
with operators/companies and the authority's inspectors who oversight the sector. Workshops are held with sector stakeholders to identify risks and explore potential areas for improvement to minimise the current likelihood, consequences or both for the identified risks. Safety improvements may include reviewing regulation, safety promotion campaigns or an emphasis on improved supervision and mentoring. Finally the SRP risk register which contains risks and actions, ownership of actions, a timeline and an action plan is communicated to sector members. The exchange of safety performance information is important to update the risk profile and trigger a review.

In order for the SRP to be a living profile, it is desirable to map the sector risk register with sector entities' risk registers to obtain safety assurance for sector participants operations. By extending the mapping to the scope and criteria for the surveillance task, the safety surveillance program can be directed to review risks and assess the effectiveness of risk controls within the sector. As the SMS of an operator will be a repository for incident reporting, analysis and investigation, gaps in relation to the sector risk register and the sector entity's risk register will be captured and current risk controls if inadequate may be enhanced to better address the risks.

The sector risk register can be used to develop a set of SPIs to monitor safety performance. Information on incidents from sector entities' risk registers will be used to reports trends in the sector's safety performance and through annual sector safety forums additional information will be captured to make the SRP a living profile. The key to a successful SRP process is good collaboration and engagement with all stakeholders as evidenced in the workshops conducted by CASA.

\subsection{SRP Process}

The SRP process contains three phases (Figure 1). Phase 1 establishes the sector's context and current state through data collection, fusion and analysis. A workshop is conducted towards the end of Phase 1 with participants who include sector operators, safety regulator and other sector stakeholders who provide infrastructure and support services to sector participants. Key outputs from the workshop are a list of sector objectives, a SWOT (Strengths, Weaknesses, Opportunities and Threats) analysis which assists with the identification of hazards and the associated risks for the sector.

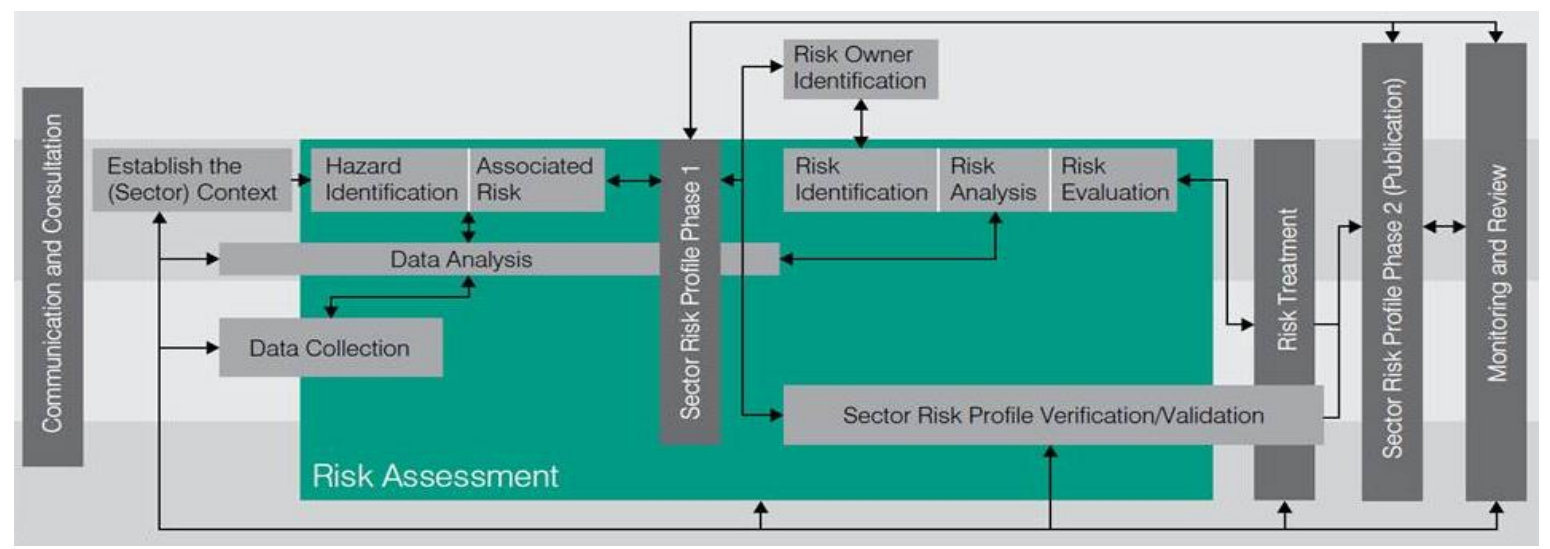

Figure 1. SRP process (CASA, 2016a) with Phase 3 embedded in the 'Monitoring and Review' stage.

The principal task in SRP Phase 2 is to transform the hazard table into a risk register. The Phase 2 workshop proceedings are conducted by collaborating and engaging with a small group of sector participants to develop a list of risks for the sector. Workshop participants initially draft a precise risk statement for each risk influencing sector operations. The participants key tasks are to identify risk causes, impacts and existing risk controls for the risk, along with an evaluation of the current likelihood, most credible (rather than the worst case) impact/consequence and risk rating for the risk. If the rating is outside the ALARP (As Low As Reasonably Practicable) limit, additional risk treatments are identified with accountability assigned to the owners best placed to treat the risk. The residual risk is assessed assuming these treatments are in place. Following the workshop, sector participants are provided with an opportunity to review and comment on the risk register.

The implementation of the sector risk register forms Phase 3 of the SRP process and this entails mapping the sector risk register to sector entities' risk registers to provide a level of safety assurance to the safety authority. SPIs are also developed for the sector to measure and monitor the sector's safety performance level. Safety trend analysis and trend reporting forms an important part of Phase 3 and a risk review may be triggered within a defined period to complete the feed-back loop of risk management suggested by ISO 
Lin et al., Introduction to sector risk profile methodology with application to civil aviation sectors

31000:2009. The sector risk register is also integrated into an individual operator's SMS and into authority's safety surveillance program. The elements of Phase 3 attempt to ensure the SRP is a living profile.

\subsection{SRP in a big picture and the outputs of SRP}

SRP can be used by sector entities and an oversighting authority in several ways. The greatest value of a SRP is derived when participants, including the authority, read the risk statements, decide which ones apply to their organisation and then determine what they can do to minimize that risk. By addressing individual elements of risk within a sector, the overall accident rate and costs to the sector can be reduced. SRPs also help to inform the authority where to target its safety oversight activity and resources.

As shown in Figure 2, once the risk profile is implemented by adopting the measures described earlier, SRP becomes fully embedded in a sector entity's SMS and informs amendments or additions to rule-making, additional safety promotion initiatives and the effectiveness of the authority's safety oversight program.

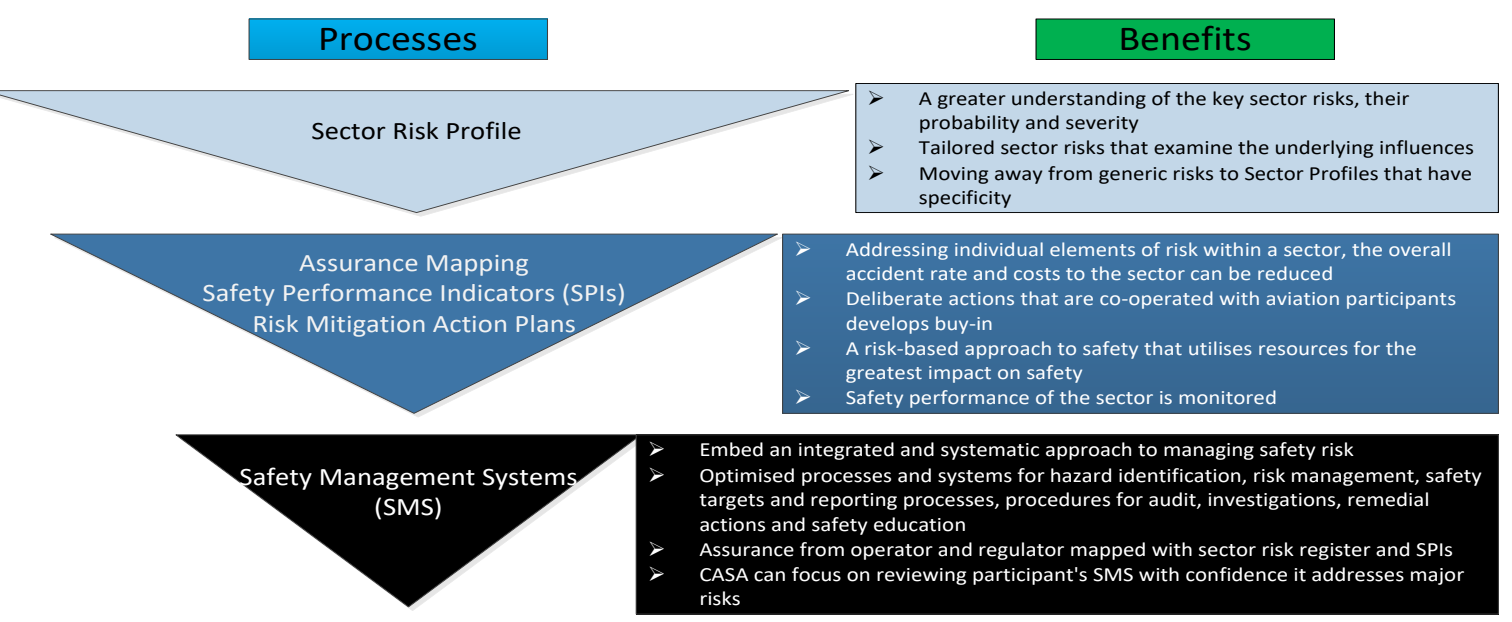

Figure 2. SRP in a big picture.

The outputs generated from the SRP will assist authorities, sector entities and primary stakeholders to:

- identify and understand safety risks,

- ensure relevant risks are incorporated in an entity's SMS,

- identify system vulnerabilities which can inform the authority where to target its resources,

- $\quad$ reduce safety risk within sector, and

- maintain confidence of the public and key stakeholders.

The way forward in the 'SRP space' is to 'collaborate more' and 'engage more' with all stakeholders to increase understanding, promote safety improvement opportunities, and work on solutions together. This is underpinned by creating a culture that encourages open and honest communication, listening to others, showing respect and maintaining trust.

\section{CASE STUDY}

\subsection{Rationale}

Civil aviation has a high degree of diversity in terms of aircraft activity, aircraft types and the infrastructure and services used to support aircraft activity. One way of addressing diversity is by creating sectors based on aircraft activity with sectors aligned with specific regulations. As a result, Australia's civil aviation has been classified as 12 primary sectors for flying operations grouped in three segments: air transport, aerial work and general aviation. Aerodromes, air traffic services, flight training organizations and maintenance organizations are support sectors that support flying operations (CASA, 2015).

Australian domestic small aeroplane transport sector relates to the carriage of passengers, their accompanying baggage and/or freight using fixed-wing aircraft with up to nine passenger seats and with the maximum takeoff weight below $8,618 \mathrm{~kg}$ (or 19,000 pounds). This sector is essential to the social, economic and cultural sustainability of Australia's remote and regional communities with over 4 million regional Australians rely on regional air services (RAAA, 2011). The sector has about 180 air operator certificate (AOC) holders using 
Lin et al., Introduction to sector risk profile methodology with application to civil aviation sectors

about 760 small aeroplanes and provides an annual average of 380,000 flight hours separated by the scheduled or regular service (14\%) and the non-scheduled or charter services (86\%) (CASA, 2016a).

During the period of 2006 to 2014, there were a total of 115 accidents in the sector including seven fatal accidents resulting in nine fatalities. The accident rate of the sector was 33.7 accidents per million flight hours compared to 0.9 accident per million flight hours from the Australian domestic large aeroplane (98+ seats) transport sector (CASA, 2016b). Clearly there is much room to improve the sector's safety performance.

\subsection{Phase 1 process}

The risk profiling process had a strong spine of consultation and peer review and was supported by CASA and industry risk teams reviewing a variety of datasets and surveys and applying quantitative and qualitative analytical tools to develop a list of hazards and associated risks for small aeroplane transport operations.

Up to 10 participants from the sector were invited to attend both SRP workshops including AOC holders, chief pilots, maintenance engineers, safety managers and an aviation association. SMEs including flight inspectors, airworthiness inspectors, safety system inspectors and aviation regulation developers/writers (standards officers) formed a CASA reference group.

In the first SRP workshop, participants reviewed the current state of the sector (CASA, 2016a) which was prepared by CASA based on data fusion (Figure 3) and analysis and also included sector's safety surveillance findings. Participants then developed a list of sector objectives (Table 1) which was later used in the development of the risk register. Following that, a SWOT analysis was performed which led to the development of a hazard list related to the sector's operations. Associated risks for each hazard were identified through a workshop facilitation process. For example, a hazard might be wildlife in vicinity of aerodrome/airstrip and the associated risk would be aircraft collision with wildlife.

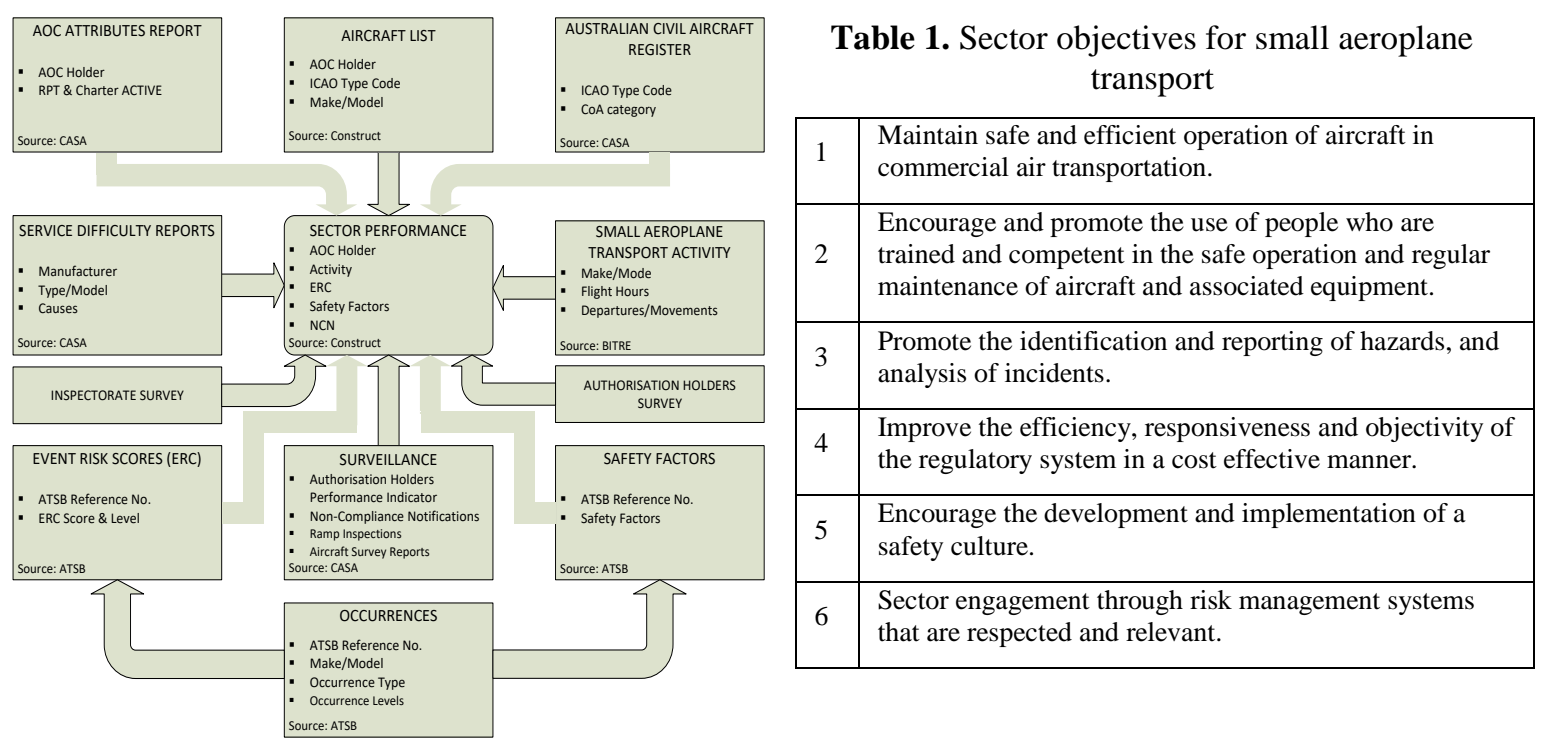

Figure 3. Architecture of information fusion.

\subsection{Phase 2 process}

The first task in Phase 2 was using the hazard list to develop risk statements. To develop these statements, the associated risks were grouped to form a collection of risks which had a degree of affinity with the original hazard which then emerged as the cause/source of that risk. For the previous example, collision with wildlife is a risk for the sector and the causes/sources of this risk are those hazards associated with this risk, namely, wildlife in vicinity of aerodromes, seasonal/migratory bird movement, night operations, etc. Note that the sector's threats and weaknesses from the SWOT analysis also supplemented the risk list. Each derived risk was presented for risk evaluation and validated in the second workshop.

The principal task in the second SRP workshop was to develop and populate the risk register for the sector. In order to perform this task, a six-by-six risk matrix (Figure 4) applicable to the sector was developed. Note that it is important to design a risk matrix with the same increase/decrease system, such as a 10-fold system (Lin and Jarrett, 2010) both in the likelihood and consequence descriptors, such that the resulting risk rating values will be symmetrical in relating to the top right corner of the matrix. That is, the risk of the likelihood 4 
Lin et al., Introduction to sector risk profile methodology with application to civil aviation sectors

with consequence 3 is equivalent to the risk of the likelihood 3 with consequence 4 . Note that an extra character (i.e. a to e) was also assigned to the risk rating to provide backwards transparency.

For each risk, the workshop participants collaboratively populated the risk causes/sources, impacts, stakeholders, primary risk owner, current risk controls, control ownership, current likelihood of the most credible event, current consequence and current risk rating. When the risk rating was above the ALARP limit, further risk treatments were also identified including the treatment owners. Then the residual likelihood and consequence were further evaluated assuming those treatments are in place. Finally, the residual risk rating was obtained for the risk, the sector objectives impacted by the risk were identified and a future review date was assigned; see an example in Figure 5.

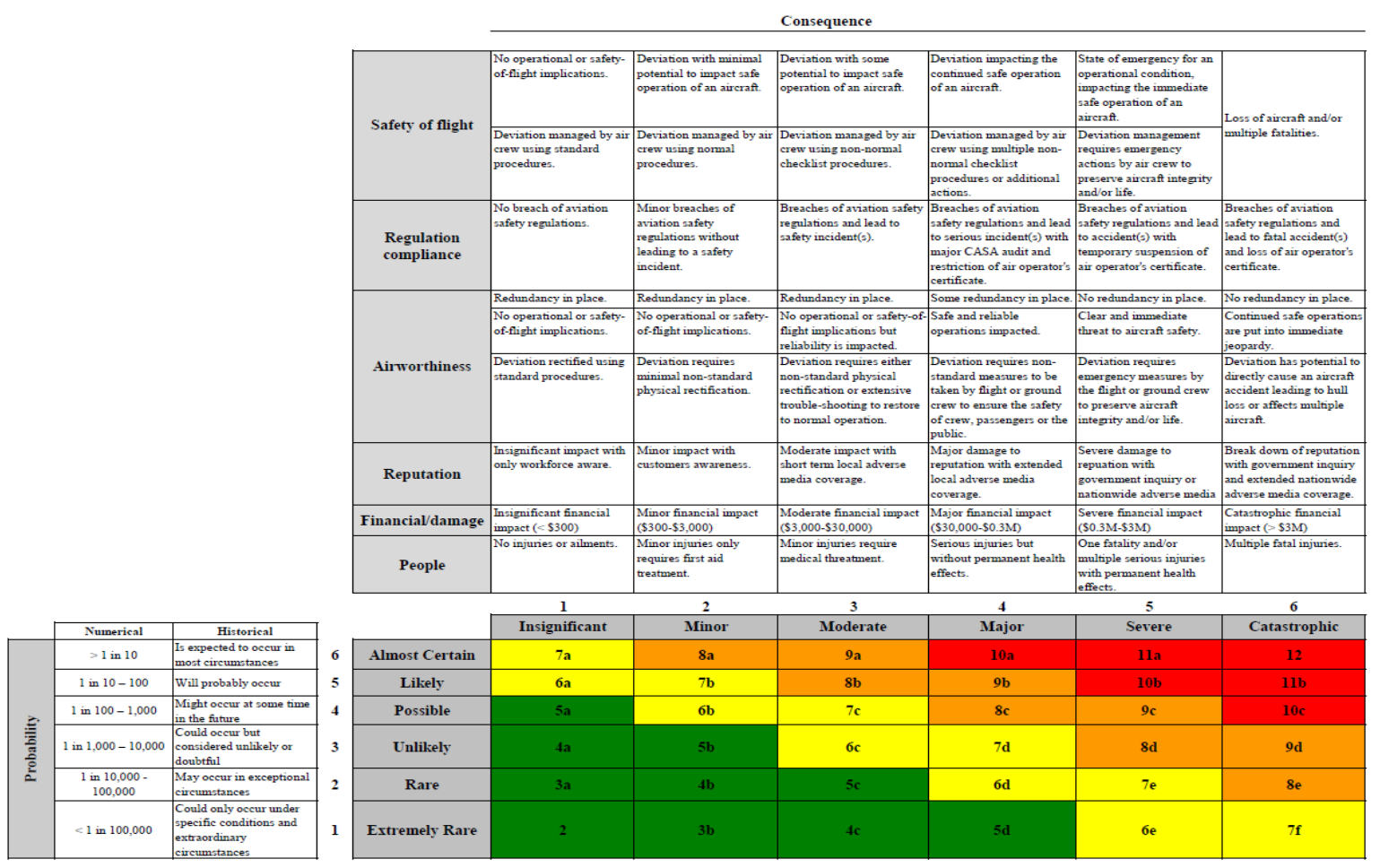

Figure 4. Risk matrix developed for the small aeroplane transport sector.

\begin{tabular}{|c|c|c|c|c|c|c|c|}
\hline Risk & \begin{tabular}{|c|} 
Risk Owner \\
- Primary \\
\end{tabular} & Current Controls & \begin{tabular}{|r|} 
Current \\
Rating \\
\end{tabular} & Treatment Description & Treatment Owner & $\begin{array}{l}\text { Residual } \\
\text { Risk }\end{array}$ & $\begin{array}{c}\text { Risk Review } \\
\text { Date }\end{array}$ \\
\hline \multirow{2}{*}{$\begin{array}{l}\text { Collision with } \\
\text { wildlife in } \\
\text { flight }\end{array}$} & \multirow[b]{2}{*}{$\begin{array}{l}\text { Aircraft } \\
\text { operator }\end{array}$} & \multirow{2}{*}{$\begin{array}{l}\text { Aerodrome wildlife } \\
\text { management plan } \\
\text { Aerodrome } \\
\text { reporting to } \\
\text { operators } \\
\text { Flight crew training }\end{array}$} & \multirow[b]{2}{*}{$\begin{array}{l}\text { Medium } \\
\quad 7 c\end{array}$} & $\begin{array}{l}\text { Improve reporting from aerodrome reporting officer (ARO) through } \\
\text { effective Wildlife Management Plan }\end{array}$ & Aerodrome owner & \multirow{2}{*}{$\begin{array}{l}\text { Medium } \\
6 c\end{array}$} & \multirow[b]{2}{*}{2017} \\
\hline & & & & Develop whole-of-government approach towards aerodrome hazards & $\begin{array}{l}\text { CASA/ Dept of Infrastructure / } \\
\text { Airservices Australia }\end{array}$ & & \\
\hline
\end{tabular}

Figure 5. Simplified version of a risk example for the sector.

Note that if a particular sector objective was not impacted by any risks, it alerts participants that either this objective was not relevant to the sector's safety operations or there may be risks to the sector that have not been identified.

Also note that the statistics of the sector's safety related occurrences collected by Australian Transport Safety Bureau (ATSB) might be used to estimate the current likelihood of a risk; however, workshop participants' opinions based on their operational knowledge, records and experience were the principal source of the evaluation. This is an advantage of SRP based on industry workshops versus other risk assessments performed by risk practitioners who may lack operational knowledge and experience.

The draft risk register compiled in the second workshop was further validated in a series of mini workshops with CASA staff and within the small aeroplane transport sector held across CASA's regional offices using video communication facilities. Furthermore, a public document of the small aeroplanes SRP (CASA, 2017) including a simplified version of the final risk register was also developed for the interests of the community at large. 
Lin et al., Introduction to sector risk profile methodology with application to civil aviation sectors

\subsection{Phase 3 process}

The implementation and integration of the sector risk register are to be carried out in three stages within Phase 3:

1. Assurance mapping of the sector risk register with a sector entity's risk register in terms of risks identified, current risk controls/mitigations in place;

2. Assurance mapping of the CASA surveillance scope and criteria to seek assurance that the surveillance program is targeting the risks in the sector and evaluating the effectiveness of risks controls/mitigations in addressing risks; and

3. Implementation of risk treatments by treatment owners.

When the sector risk register is fully implemented, any additional risk treatments identified in the risk register become risk controls and thus reduce the safety risk level of the sector. The responsibility for implementation of the treatment measures for which sector has accountability rests with AOC holders, operators and pilots.

\section{CONCLUSION}

Sector risk profiling (SRP) methodology and process which follows the framework of ISO 31000:2009 was presented. The main advantage of SRP is that the process is consultative with sector's subject matter experts which is assisted with a desktop analysis of relevant and appropriate data. Furthermore, the implementation and integration of the sector risk register to the safety surveillance program and a sector entity's risk register makes the profile a living profile. The main output of the SRP is a sector risk register where each risk is evaluated in association with the existing risk controls. When a particular risk is assessed as outside the ALARP limit, further risk treatments are identified to reduce the risk severity and the owners for these treatments are identified. Based on the risk register, safety performance indicators can also be developed for the sector to measure and monitor the sector's safety performance. The case study of Australia's small aeroplane transport sector SRP has demonstrated the application of the SRP process. SRP forms an important building block of Australia's Aviation State Safety Program.

\section{REFERENCES}

CASA (2015). Australian aviation community sectors. Civil Aviation Safety Authority (CASA), Canberra, Australia, https://www.casa.gov.au/files/australian-aviation-community-sectors, accessed on the $20^{\text {th }}$ of September 2017.

CASA (2016a). State of small aeroplane transport sector report. CASA, Canberra, Australia.

CASA (2016b). State of large aeroplane transport sector report. CASA, Canberra, Australia.

CASA (2017). Sector risk profile for the small aeroplane transport sector. CASA, Canberra, Australia, https://www.casa.gov.au/files/srpsmallaerobookletpdf, accessed on the $20^{\text {th }}$ of September 2017.

Chapman, R.J. (2011). Simple tools and techniques for enterprise risk management. Wiley Finance.

Infrastructure (2016). Australia's Aviation State Safety Programme. Department of Infrastructure and Regional Development, Canberra, Australia, https://infrastructure.gov.au/aviation/safety/ssp/index.aspx, accessed on the $20^{\text {th }}$ of September 2017.

ISO 31000:2009. Risk management - Principles and guidelines. The International Organization for Standardization.

Lin, X.G. and Jarrett, R. (2010). A Practical Approach to Multi-Hazard Risk Assessment. Joint Statistical Meetings (JSM 2010), Vancouver, Canada, 31 July - 5 August, 456-470.

RAAA (2011). Regional Aviation Association of Australia (RAAA) submission to the Australian Productivity Commission Economic Regulation of Airport Services Public Inquiry, http://www.pc.gov.au/inquiries/completed/airport-regulation/submissions/sub049.pdf, accessed on the $20^{\text {th }}$ of September 2017.

UK (2016). Sector risk profile 2016. Homes \& Communities Agency, UK, https://www.gov.uk/government/uploads/system/uploads/attachment_data/file/554083/Sector_Risk_Profil e_2016.pdf, accessed on the $20^{\text {th }}$ of September 2017. 